\title{
Correlation between red blood cell transfusion volume and mortality in patients with massive blood transfusion: A large multicenter retrospective study
}

\author{
JIANG-CUN YANG $^{1 *}$, YANG SUN $^{1 *}$, CUI-XIANG XU ${ }^{2}$, QIAN-LI DANG ${ }^{3}$, \\ LING $\mathrm{LI}^{4}$, YONG-GANG XU ${ }^{5}$, YAO-JUN SONG ${ }^{1}$ and HONG YAN ${ }^{6}$ \\ ${ }^{1}$ Department of Transfusion Medicine, ${ }^{2}$ Shaanxi Provincial Center for Clinical Laboratory and \\ Departments of ${ }^{3}$ Dermatology, ${ }^{4}$ Laboratory and ${ }^{5}$ Urology, The Third Affiliated Hospital of \\ Medical College of Xi'an Jiaotong University; ${ }^{6}$ Department of Epidemiology and Health Statistics, \\ Medical College of Xi'an Jiaotong University, Xi'an, Shaanxi 710061, P.R. China
}

Received April 4, 2014; Accepted October 23, 2014

DOI: $10.3892 / e t m .2014 .2068$

\begin{abstract}
This study aimed to explore the correlation between red blood cell (RBC) transfusion volume and patient mortality in massive blood transfusion. A multicenter retrospective study was carried out on 1,601 surgical inpatients who received massive blood transfusion in 20 large comprehensive hospitals in China. According to RBC transfusion volume and duration, the patients were divided into groups as follows: 0-4, 5-9, 10-14, 15-19, 20-24, 25-29, 30-39 and $\geq 40$ units within 24 or $72 \mathrm{~h}$. Mortality in patients with different RBC transfusion volumes was analyzed. It was found that patient mortality increased with the increase in the volume of RBC transfusion when the total RBC transfusion volume was $\geq 10$ units within 24 or $72 \mathrm{~h}$. Survival analysis revealed significant differences in mortality according to the RBC transfusion volume $\left(\chi^{2}=72.857\right.$, $\mathrm{P}<0.001)$. Logistic regression analysis revealed that $\mathrm{RBC}$ transfusion volume is an independent risk factor [odds ratio $(\mathrm{OR})=0.52$; confidence interval $(\mathrm{CI})$ : 0.43-0.64; $\mathrm{P}<0.01$ ] for the mortality of patients undergoing a massive blood transfusion. When RBCs were transfused at a volume of 5-9 units within 24 and $72 \mathrm{~h}$, the mortality rate was the lowest, at 3.7 and $2.3 \%$ respectively. It is concluded that during massive blood transfusion in surgical inpatients, there is a correlation between $\mathrm{RBC}$ transfusion volume within 24 or $72 \mathrm{~h}$ and the mortality of the patients. Patient mortality increases with the increase in the volume of RBC transfusion. RBC transfusion volume, the
\end{abstract}

Correspondence to: Dr Hong Yan, Department of Epidemiology and Health Statistics, Medical College of Xi'an Jiaotong University, 76 Yanta West Road, Xi'an, Shaanxi 710061, P.R. China

E-mail: hongyanyh@sina.cn

*Contributed equally

Key words: massive blood transfusion, red blood cell, mortality, retrospective analysis, multicenter length of stay at hospital and intensive care unit stay constitute the independent risk factors for patient mortality.

\section{Introduction}

Massive blood transfusion is commonly defined as the administration of $\geq 10$ units of packed red blood cells ( $\mathrm{pRBCs)}$ to an individual patient $(1,2)$ or the transfusion of more than one blood volume in $24 \mathrm{~h}$ (1,3-5). Alternative definitions include a $\geq 50 \%$ loss in blood volume within $3 \mathrm{~h}$ or a rate of loss of $150 \mathrm{ml} \mathrm{blood} / \mathrm{min}$ in the severe traumatic and emergent situations (3). Massive blood transfusion is often provided to those who are injured during military operations, who have multiple injuries due to other causes, and who undergo complex surgery. A rational blood transfusion can improve the outcome of surgery, whereas unreasonable transfusion can increase mortality in patients.

Transfusion plays a key role in saving the lives of patients who have suffered massive blood loss. However, studies have found that mortality remains high for trauma patients who have received massive blood transfusion and suggest that there is a certain correlation between RBC transfusion volume and the mortality of patients (6-8). Stanworth et al (9) found that the mortality of patients who had received a pRBC transfusion was $9 \%$ for $0-5$ units, $22 \%$ for $6-9$ units and $42 \%$ for $\geq 10$ units. Thus, it is necessary to maintain a balance between the advantages and disadvantages of RBC transfusion during massive blood transfusion.

Therefore, a multicenter retrospective study was conducted on cases of massive blood transfusion in 20 comprehensive hospitals from different regions of China to explore the correlation between $\mathrm{RBC}$ volume and the mortality of surgical inpatients with massive blood transfusion.

\section{Materials and methods}

Study protocol. This study was retrospective in nature. Data were collected from the medical records of surgical inpatients who received massive transfusion at 20 large-scale hospi- 
tals between January 2009 and December 2010. Between June 2010 and January 2011, 2,000 copies of the Massive Transfusion Survey Table (hereafter referred to as the Survey Table) were distributed to 20 Class III comprehensive hospitals in the northwest, southwest, central south, north and northeast regions of China. Members of the National Massive Transfusion Current Status Investigation Coordination Group (hereafter referred to as the Coordination Group) were responsible for collecting the data from these hospitals using the Survey Table. The data analysis was conducted at Shaanxi Provincial People's Hospital, which is the Third Affiliated Hospital of the Medical College of Xi'an Jiaotong University (Xi'an, China). The present study was approved by the ethics committee of Xi'an Jiaotong University.

Study population. Patients who received a transfusion of $\geq 10$ units of $\mathrm{pRBCs}$ over a period of $\leq 24 \mathrm{~h}$ for trauma, cardiac surgery, obstetric conditions or other common surgeries (for example, orthopedic, thoracic, general, urinary, hepatobiliary and neurological surgery) were included in the study. By contrast, patients with coagulation disorders, hepatic failure due to medical causes, and coagulopathies were excluded from the analysis. Patients who received transfusions of $<10$ units for $\leq 24 \mathrm{~h}$ were assigned to the control group. Informed consent was obtained from the patients or the patients' families prior to their inclusion in the current study.

Survey table. The directors of the transfusion departments of the 20 participating hospitals discussed the topic, consulted experts and designed the Survey Table with reference to several international and domestic sources, in accordance with the principles of equality, voluntariness and mutual benefits. A meeting of the Coordination Group was then held, where 35 experts of clinical transfusion, surgery, anesthesia, gynecology and obstetrics, hematology and medical statistics discussed the study protocol and mode of data collection and also perfected and added supplements to the Survey Table. Suitable training was then offered to the investigating staff.

Components of the survey table. The survey Table comprised four sections, as follows: i) Clinical and demographic characteristics of the patient, including name, gender, age, body weight, blood type, ethnicity, admission number, admission department, primary diagnosis, secondary diagnosis, pathologic diagnosis, nature of surgery and vital signs on admission. ii) Details regarding the perioperative complications, clinical condition within $24 \mathrm{~h}$ and after $24 \mathrm{~h}$ of the transfusion, and the total amount of blood transfused. iii) The results of the following blood tests performed before, within $24 \mathrm{~h}$ and after $24 \mathrm{~h}$ of transfusion: routine blood test, coagulation tests, liver function test, kidney function test, and arterial blood gas analysis. iv) Adverse events due to massive transfusion.

Quality control. The Survey Table was first subjected to a small-scale preliminary test at Shaanxi Provincial People's Hospital so that revisions could be made on the basis of the results and comments by experts to further improve the Table. One unit of pRBCs was derived from $200 \mathrm{ml}$ whole blood and had a volume of 140-172 ml. One unit of fresh frozen plasma (FFP) was derived from $200 \mathrm{ml}$ whole blood and had a volume of $100 \mathrm{ml}$. One bag of apheresis platelet was 10 units, and had a volume of 150-250 ml. One unit of platelet concentrate was derived from $200 \mathrm{ml}$ whole blood and had a volume of 20-30 ml. The pRBCs were stored at $2-6^{\circ} \mathrm{C}$. FFP was stored at $\leq-18^{\circ} \mathrm{C}$ and thawed in a $37^{\circ} \mathrm{C}$ water bath, for $\sim 10-15 \mathrm{~min}$. Platelets were stored at $20-24^{\circ} \mathrm{C}$ in a platelet shaker.

Data collected with devices and reagents. The main test devices and reagents used were as follows: Sysmex XE-2100 or XT-1800i hematology analyzer (Sysmex Corporation, Kobe, Japan); Coulter LH780 Coulter Hematology Analyzer (Beckman Coulter, Brea, CA, USA); Hitachi 7170A or 7180 Biochemical Analyzer (Hitachi, Tokyo, Japan); Roche Modular DP Automatic Biochemical Analyzer (Roche Diagnostics, Indianapolis, IN, USA); Olympus AU640 Biochemical Analyzer, (Olympus Corporation, Tokyo, Japan); Radiometer ABL-77 Blood Gas Analyzer (Radiometer, Copenhagen, Denmark); Roche Cobas- B123 Blood Gas Analyzer (Roche Diagnostics); and Sysmex CA1500/CA7000 Automatic Blood Coagulation Analyzer (Sysmex Corporation). All test reagents used were device-supporting reagents.

Data on the blood tests performed were collected from the laboratory records. These included: blood routine, coagulation tests, liver function test, kidney function and blood gas analysis. The data were collected for the blood tests performed prior to transfusion and at 16 different units during the 24-h transfusion $(2 \mathrm{U}, 4 \mathrm{U}, 6 \mathrm{U}, 8 \mathrm{U}, 10 \mathrm{U}, 12 \mathrm{U}, 14 \mathrm{U}, 16 \mathrm{U}, 18 \mathrm{U}, 20 \mathrm{U}$, $22 \mathrm{U}, 24 \mathrm{U}, 26 \mathrm{U}, 28 \mathrm{U}, 30 \mathrm{U}$ and $40 \mathrm{U}$ ) and subjected to statistical analysis. The tests were conducted at the laboratory of each participating hospital, each of which undergoes internal quality control and an external quality assessment conducted by the Clinical Test Center of the Ministry of Health.

Statistical analysis. Statistical analysis was conducted using SPSS software, version 18.0 (SPSS, Inc., Chicago, IL, USA). Epidata version 3.01 (Epidata Association, Odense, Denmark) was used for double data entry verification and database construction. The data on the demographic characteristics and clinical features were expressed as means with standard deviations or as absolute numbers. Categorical variables were analyzed by $\chi^{2}$ test, while continuous variables with normal distribution were analyzed by the Shapiro-Wilk test, analysis of variance, or the Kruskall-Wallis test, as appropriate. The Bonferroni method was applied for post-hoc tests to determine the significance of the differences between the group that received massive transfusion and the control group that did not. Linear regression was used to describe the relation between the number of units of pRBCs transfused and the platelet count. $\mathrm{P}<0.05$ was considered to indicate a statistically significant result.

\section{Results}

Survey results. In total, 1,753 copies of the Survey Table were received from the 20 hospitals and the recovery rate was $87.65 \%(1,753 / 2,000)$. Of these, 1,601 copies were qualified tables without missing items and the qualification rate was $91.33 \%(1,601 / 1,753)$. The demographics and clinical data for the various RBC transfusion volume groups are shown in Table I. Among the 1,601 massive blood transfusion 


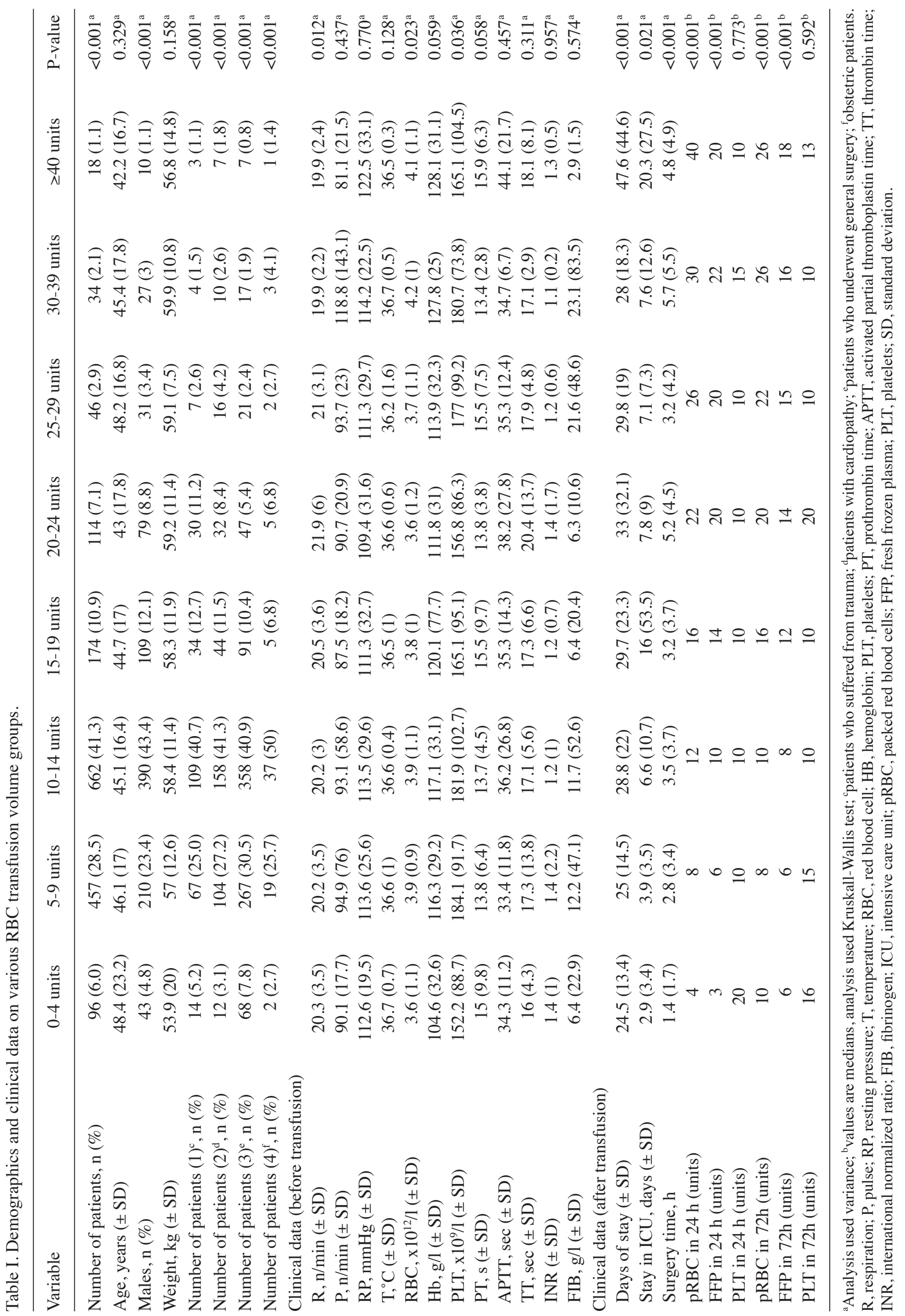


patients, 268 patients had undergone trauma (mortality, 34; survival, 234; mortality rate, 12.69\%), 383 patients had undergone cardiac surgery (mortality, 53; survival, 330; mortality rate, $13.84 \%), 876$ patients had undergone general surgery (mortality, 42; survival, 834; mortality rate, 4.79\%) and 74 patients were obstetric patients (mortality, 3; survival, 71; mortality rate, $4.05 \%)$.

Patient mortality. The mortality of the patients increased with the increase in the volume of RBC transfusion when the total RBC transfusion was $>10$ units, regardless of whether this was within 24 or $72 \mathrm{~h}$. Within $24 \mathrm{~h}$, as the volume of transfused RBCs increased from 10 to 40 units, the mortality rate rose from 6.0 to $38.9 \%$. Within $72 \mathrm{~h}$, as the volume of RBCs increased from 10 to 40 units, the mortality rate rose from $5.2 \%$ to $28.0 \%$. When the volume of transfused RBCs was 5-9 units within 24 and $72 \mathrm{~h}$, the mortality rate was the lowest, which was 3.7 and $2.3 \%$, respectively. For transfusion with $0-4$ units, the mortality rates were 7.3 and $9.7 \%$, respectively (Table II).

Survival analysis showed that there were significant differences in mortality among the patients according to the RBC transfusion volume $\left(\chi^{2}=72.857, \mathrm{P}<0.001\right.$; Fig 1$)$.

Logistic regression analysis. Multivariate logistic regression analysis was performed with hospital mortality as the dependent variable. The following variables were considered as independent predictors: i) age, ii) gender, iii) surgery duration, iv) weight, v) length of stay in hospital, vi) intensive care unit (ICU) stay, vii) RBC volume (in $24 \mathrm{~h}$ ) and viii) FFP volume (in $24 \mathrm{~h}$ ). The results are presented as odds ratios (ORs) with $95 \%$ confidence intervals $(95 \% \mathrm{CI}$; Table III). The factors that were identified to be significantly correlated with mortality were $\mathrm{RBC}$ volume $(\mathrm{OR}=0.52,95 \% \mathrm{CI}: 0.43-0.64 ; \mathrm{P}<0.001)$, length of stay in hospital $(\mathrm{OR}=2.79 ; 95 \% \mathrm{CI}: 1.31-5.92 ; \mathrm{P}=0.01)$, and ICU stay $(\mathrm{OR}=0.43 ; 95 \% \mathrm{CI} ; 0.21-0.88 ; \mathrm{P}=0.02)$.

\section{Discussion}

Transfusion plays an important role in saving the lives of patients in emergency and danger. Timely and sufficient blood transfusion is critical for the survival of patients who have suffered massive blood loss. Survival rates following massive transfusion have significantly increased in recent years. However, massive transfusion protocols have not always been associated with improved mortality (10). Long et al (11) examined the impact of postoperative hematocrit as an indicator of survival following massive transfusion in the trauma patient. They found that transfusion to hematocrits between 29.1 and $39 \%$ conveyed a survival benefit, whereas resuscitation to supraphysiologic hematocrits $\geq 39 \%$ conveyed no additional survival benefit. Sharpe et al (12) evaluated the effect of the number of RBC units transfused on the plasma:RBC and platelet:RBC ratios and their association with mortality in patients receiving massive transfusion. The authors found that patients receiving relatively higher quantities of RBCs were more likely to have a lower plasma:RBC ratio and were more likely to die.

The present study found that among 1,048 patients who received $\geq 10$ units $R B C$ transfusion volume within $24 \mathrm{~h}$, 
Table III. Results of multivariate logistic regression analysis.

\begin{tabular}{lccccc}
\hline Variable & $\beta$ & SE & P-value & Odds ratio & $95.0 \%$ CI \\
\hline RBC volume & -0.65 & 0.10 & $<0.001$ & 0.52 & $0.43-0.64$ \\
Length of stay & 1.02 & 0.38 & 0.01 & 2.79 & $1.31-5.92$ \\
ICU stay & -0.84 & 0.36 & 0.02 & 0.43 & $0.21-0.88$ \\
Constant & 2.14 & 1.00 & 0.03 & 8.48 &
\end{tabular}

SE, standard error; CI, confidence interval; RBC, red blood cell; ICU, intensive care unit.

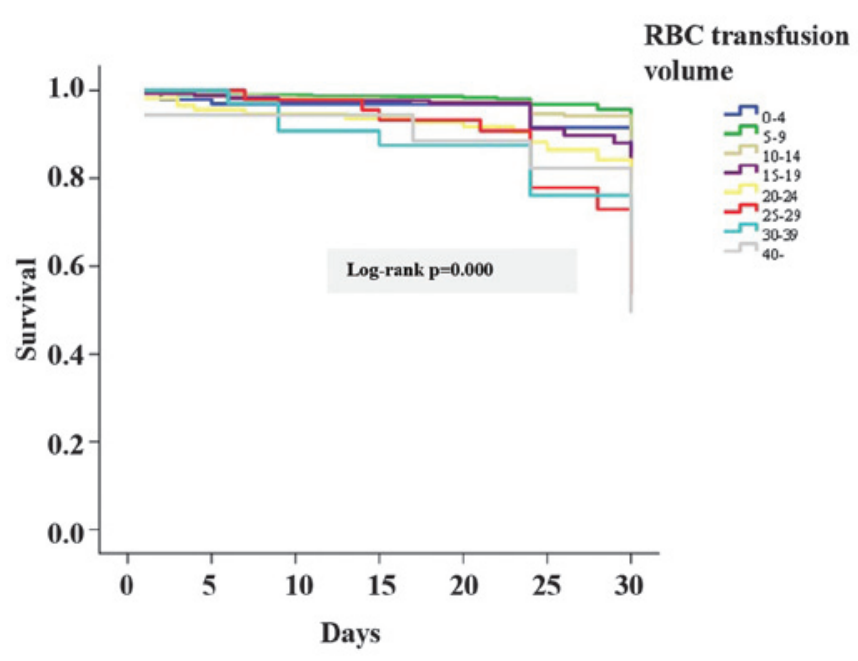

Figure 1. Kaplan-Meier survival chart of different red blood cell (RBC) transfusion volume groups.

the mortality rate was $10.31 \%$, which is lower than the rate observed in related studies $(9,13)$. This may be because the 20 medical institutions that participated in the present study are large general hospitals with better conditions, or because fewer trauma cases and a greater proportion of general surgery cases with good pre-operation preparation were included in this study, or due to the immediate application of fresh frozen plasma with a high percentage accompanying the RBC transfusion to correct coagulation at an initial stage; further research is required to clarify this. The present study also found that if the patients were classified by clinical department, the mortality of cardiac surgery and trauma patients receiving massive blood transfusion was 13.84 and $12.69 \%$ respectively, which is higher than the 4.79 and $4.05 \%$ mortality of general surgery and obstetrics patients, respectively.

According to previous studies, there is some correlation between RBC transfusion volume and the mortality of patients. The study conducted by Como et al (8) demonstrated that the mortality rate of 147 patients with massive blood transfusion was up to $39 \%$ and the mortality rate was $51 \%$ for patients receiving $>50$ units of blood products transfused within $24 \mathrm{~h}$. Stanworth et al (9) indicated that the mortality rate was $9 \%$ when patients receiving $0-5$ units pRBC transfusion, $22 \%$ for those receiving 6-9 units pRBCs and $42 \%$ for those receiving $\geq 10$ units pBRCs. Surgenor et al (13) reported that RBC transfusion during or following cardiac surgery showed a certain correlation with the increase in the mortality of patients. The long-term risk of mortality was $16 \%$, which was higher than that of patients undergoing transfusion with 1 or 2 units of pRBCs. The results of the present study for 1,601 surgical inpatients with transfusion were consistent with these previous studies. The present study also identified that a correlation existed between RBC transfusion volume and patient mortality. The multivariate logistic regression analysis results indicated that RBC transfusion volume (in $24 \mathrm{~h}$ ), length of stay and ICU stay constitute independent risk factors for patient mortality.

However, certain limitations existed in this study. It was a large multicenter retrospective study, with a review of registry data in which a variable proportion of records may have been missing data. This is inevitable to a degree in analyses of multiple registries.

In summary, the present study highlights the correlation between RBC transfusion volume and patient mortality for surgical inpatients with massive blood transfusion. The mortality rate increased with as the volume of RBC transfusion increased. RBC transfusion volume, length of stay and ICU stay constitute independent risk factors for patient mortality during massive blood transfusion.

\section{Acknowledgements}

This study was supported by a grant from Johnson \& Johnson (China) Medical Equipment Co., Ltd (Shanghai, China). The authors would like to thank the other 19 centers participating in this study: Shi-Jie Mu, Ai-Jun Xia and Xian-Qin Zhang from Xijing Hospital, the Fourth Military Medical University (Xi'an, China); Dai-Yu Li from Affiliated Hospital of Luzhou Medical College (Luzhou, China); Shu-Min Zhao from Southwest Hospital, the Third Military Medical University (Chongqing, China); Wei Jiao from the People's Hospital of Zhuang Autonomous Region (Nanning, China); Li Tong from the First Affiliated Hospital of Kunming Medical University (Kunming, China); Qing-Bao Meng from Shenzhen People's Hospital (Shenzhen, China); Jie Li from the Fourth Clinical Medical College of Hebei Medical University (Shijiazhuang, China); Shi-Ming Yang from Tangdu Hospital, the Fourth Military Medical University (Xi'an, China); Suo-Liang Yao from Xi'an Hong Hui Hospital (Xi'an, China); Bi-Juan Li from Xiangya Hospital of Center-South University (Changsha, China); Qiu-Shi Wang from Shengjing Hospital of China Medical University (Shenyang, China); Cui-Ying Li from General Hospital of Chengdu Military Region (Chengdu, China); Mei-Ning Han from the Second Affiliated Hospital of 
Medical College of Xi'an Jiaotong University (Xi'an, China); Zhi-Xi Hu from Yan'an University Affiliated Hospital (Yan'an, China); Jin-Shan Jiao from the First Affiliated Hospital of Shanxi Medical University (Taiyuan, China); Xian-Ping Lv from the First Affiliated Hospital of Zhengzhou University (Zhengzhou, China); Yan-Li Bai from Xi'an Central Hospital (Xi'an, China); Xiao-Xia Shi from Xianyang 215 Hospital (Xianyang, China); and Fang-Xiang Chen from Daping Hospital, the Third Military Medical University (Chongqing, China).

\section{References}

1. Malone DL, Hess JR and Fingerhut A: Massive blood transfusion practices around the globe and a suggestion for a common massive blood transfusion protocol. J Trauma 60 (6 Suppl): S91-S96, 2006.

2. Schuster KM, Davis KA, Lui FY, Maerz LL and Kaplan LJ: The status of massive blood transfusion protocols in United States trauma centers: Massive transfusion or massive confusion? Transfusion 50: 1545, 2010.

3. British Committee for Standards in Haematology; Stainsby D, MacLennan S, Thomas D, Isaac J and Hamilton PJ: Guidelines on the management of massive blood loss. Br J Haematol 135: 634-691, 2006.

4. Hewitt PE and Machin SJ: ABC of transfusion. Massive blood transfusion. BMJ 300: 107, 1990.

5. Kozek-Langenecker S: Management of massive operative blood loss. Minerva Anestesiol 73: 401-415, 2007.
6. Cinat ME, Wallace WC, Nastanski F, West J, Sloan S, Ocariz J and Wilson SE: Improved survival following massive transfusion in patients who have undergone trauma. Arch Surg 134: 964-970, 1999.

7. Riskin DJ, Tsai TC, Riskin L, Hernandez-Boussard T, Purtill M, Maggio PM, Spain DA and Brundage SI: Massive transfusion protocols: The role of aggressive resuscitation versus product ratio in mortality reduction. J Am Coll Surg 209: 198-205, 2009.

8. Como JJ, Dutton RP, Scalea TM, Edelman BB and Hess JR: Blood transfusion rates in the care of acute trauma. Transfusion 44 : 809-813, 2004

9. Stanworth SJ, Morris TP, Gaarder C, Goslings JC, Maegele M, Cohen MJ, König TC, Davenport RA, Pittet JF, Johansson PI, Allard S, Johnson T and Brohi K: Reappraising the concept of massive transfusion in trauma. Crit Care 14: R239, 2010.

10. Mitra B, O'Reilly G, Cameron PA, Zatta A and Gruen RL: Effectiveness of massive transfusion protocols on mortality in trauma: a systematic review and meta-analysis. ANZ J Surg. 83: 918-923, 2013.

11. Long K, Heaney JB, Simms ER, McSwain NE and Duchesne JC: When enough is enough: impact of packed red blood cells in massive transfusion outcomes. Am Surg 79: 810-814, 2013.

12. Sharpe JP, Weinberg JA, Magnotti LJ, Maclennan PA, Schroeppel TJ, Fabian TC and Croce MA: Accounting for differences in transfusion volume: Are all massive transfusions created equal? J Trauma Acute Care Surg 72: 1536-1540, 2012.

13. Surgenor SD, Kramer RS, Olmstead EM, Ross CS, Sellke FW, Likosky DS, Marrin CA, Helm RE, Jr, Leavitt BJ, Morton JR, Charlesworth DC, Clough RA, Hernandez F, Frumiento C, Benak A, DioData C and O'Connor GT; Northern New England Cardiovascular Disease Study Group: The association of perioperative red blood cell transfusions and decreased long-term survival after cardiac surgery. Anesth Analg 108: 1741-1746, 2009. 